Revue internationale P.M.E.

\title{
L'intention environnementale des dirigeants de PME
}

Une étude exploratoire dans l'industrie du textile-habillement

The environmental intention of company directors of SMEs

An exploratory study in the textile-clothing industry

La intención medioambiental de los dirigente de las PyMES

estudio analítico en la industria textil para la vestimenta

\section{Azzedine Tounés, Fafani Gribbaa et Karim Messeghem}

Volume 27, numéro 1, 2014

Créativité, PME et entrepreneuriat : des zones d'ombre et de lumière

URI : https://id.erudit.org/iderudit/1025692ar

DOI : https://doi.org/10.7202/1025692ar

Aller au sommaire du numéro

Éditeur(s)

Editions EMS - In Quarto SARL

ISSN

0776-5436 (imprimé)

1918-9699 (numérique)

Découvrir la revue

Citer cet article

Tounés, A., Gribbaa, F. \& Messeghem, K. (2014). L'intention environnementale des dirigeants de PME : une étude exploratoire dans l'industrie du textile-habillement. Revue internationale P.M.E., 27(1), 125-152. https://doi.org/10.7202/1025692ar
Résumé de l'article

Les recherches sur les processus comportementaux environnementaux représentent des thèmes privilégiés dans le champ de la PME. Toutefois, ils sont difficilement saisissables, car ils entrainent des changements de visions, de valeurs, d'attitudes et d'intentions. Par ailleurs, nous en savons peu sur l'intention des dirigeants de PME à l'égard de l'environnement. L'objectif de cet article est d'explorer la nature et la pertinence des relations entre l'intention environnementale des dirigeants et ses déterminants. Cette recherche s'ancre dans le champ de l'entrepreneuriat durable et mobilise la théorie du comportement planifié d'Ajzen (1991). Elle retient une perspective exploratoire à partir d'une enquête auprès de vingt dirigeants de PME du secteur du textile-habillement en Tunisie et d'une analyse lexicale. Les résultats conduisent à la formulation de huit propositions relatives aux déterminants de l'intention environnementale. Ceux-ci concernent plus précisément les conséquences attendues du comportement recherché, les actions entreprises en vue de le réaliser, les pressions des pouvoirs publics et des donneurs d'ordres étrangers, les perceptions des aptitudes environnementales et de la disponibilité des ressources, la nature de l'activité et le souhait d'imiter des modèles d'entrepreneurs.
Tous droits réservés (C Editions EMS - In Quarto SARL, 2014
Ce document est protégé par la loi sur le droit d'auteur. L'utilisation des services d'Érudit (y compris la reproduction) est assujettie à sa politique d'utilisation que vous pouvez consulter en ligne.

https://apropos.erudit.org/fr/usagers/politique-dutilisation/ 
ARTICLE - HORS THEME

\title{
Lintention environnementale des dirigeants de PME : une étude exploratoire dans l'industrie du textile-habillement
}

\begin{abstract}
Azzedine TOUNÉS
Azzedine Tounés est enseignant-chercheur à l'INSEEC Alpes-Savoie. Il est responsable du parcours "Entrepreneuriat» au sein du programme Master "Grandes Écoles ». Ses domaines de publication portent sur l'enseignement de l'entrepreneuriat, l'entrepreneur et l'apprentissage entrepreneurial dans la pensée économique et l'intention entrepreneuriale. Il a publié une dizaine d'articles dans des revues scientifiques. Il est membre du conseil d'administration de l'Académie de l'entrepreneuriat et de l'innovation.
\end{abstract}

INSEEC Alpes-Savoie 12, avenue Lac d'Annecy, Savoie Technolac 73381 Le Bourget-du-Lac-France atounes@inseec.com

Fafani GRIBAA

Gribaa Fafani est assistante d'enseignement supérieur à l'Institut supérieur de gestion de Sousse. Elle enseigne la culture entrepreneuriale. Elle est titulaire d'un doctorat en Sciences de gestion de l'Université Montpellier I et de Sousse. Elle est spécialisée en développement durable et responsabilité sociale de l'entreprise. Elle est membre du laboratoire MRM-ERFI.

Institut supérieur de gestion de Sousse Rue Abdlaaziz il Behi, BP 763 4000 Sousse - Tunisie gribaafafani@yahoo.fr

\section{Karim MESSEGHEM}

Karim Messeghem est professeur à l'Université Montpellier 1, directeur du Labex Entreprendre et responsable de l'équipe Entrepreneuriat du Laboratoire MRM. Ses travaux portent sur l'accompagnement entrepreneurial et les stratégies des PME. Vice-doyen de l'UFR AES, il codirige la chaire Jacques Cour pour l'accompagnement entrepreneurial et le Master Accompagnement entrepreneurial. Il a publié une trentaine d'articles dans des revues classées ainsi que plusieurs ouvrages dont L'Entrepreneuriat chez EMS.

Université Montpellier 1 - UFR AES

Avenue Raymond Dugrand, CS 59640

34960 MONTPELLIER CEDEX 2 - France karim.messeghem@univ-montp1.fr 


\begin{abstract}
RÉSUMÉ
Les recherches sur les processus comportementaux environnementaux représentent des thèmes privilégiés dans le champ de la PME. Toutefois, ils sont difficilement saisissables, car ils entrainent des changements de visions, de valeurs, d'attitudes et d'intentions. Par ailleurs, nous en savons peu sur l'intention des dirigeants de PME à l'égard de l'environnement. L'objectif de cet article est d'explorer la nature et la pertinence des relations entre l'intention environnementale des dirigeants et ses déterminants. Cette recherche s'ancre dans le champ de l'entrepreneuriat durable et mobilise la théorie du comportement planifié d'Ajzen (1991). Elle retient une perspective exploratoire à partir d'une enquête auprès de vingt dirigeants de PME du secteur du textile-habillement en Tunisie et d'une analyse lexicale. Les résultats conduisent à la formulation de huit propositions relatives aux déterminants de l'intention environnementale. Ceux-ci concernent plus précisément les conséquences attendues du comportement recherché, les actions entreprises en vue de le réaliser, les pressions des pouvoirs publics et des donneurs d'ordres étrangers, les perceptions des aptitudes environnementales et de la disponibilité des ressources, la nature de l'activité et le souhait d'imiter des modèles d'entrepreneurs.
\end{abstract}

\title{
MotS CLÉS
}

Entrepreneuriat durable, Intention environnementale, Responsabilité sociale des entreprises, Secteur du textile-habillement. Théorie du comportement planifié

\section{The environmental intention of company directors of SMEs : an exploratory study in the textile-clothing industry}

\begin{abstract}
Environmental behavioral processes are frequently studied in the field of SMEs. However, these processes are difficult to perceive as they involve changes in visions, attitudes and intentions. Moreover, we know little about the intention of company directors of SMEs with regard to the environment. The aim of this article is to explore the type and the pertinence of relations that exist between company directors' environmental intentions and their determining factors. This research is concerned with the field of sustainable entrepreneurship and employs Ajzen's (1991) theory of planned behavior. It presents an explorative perspective based on a survey with twenty company directors of SMEs in the textile-clothing sector in Tunisia as well as a lexical analysis. The results led to the formulation of eight propositions relating to the determining factors in environmental intention. These more precisely concern the expected results of the desired behavior, the actions undertaken with a view to achieving this, the pressure from public authorities and the foreign prime manufacturers, the perceptions of the environmental aptitudes and the availability of resources, the type of activity and the desire to imitate entrepreneurs' models.
\end{abstract}

\section{KEYWORDS}

Sustainable entrepreneurship, Environmental intention, Corporate social responsibility. Textile-clothing sector, Theory of planned behavior 


\title{
La intención medioambiental de los dirigente de las PyMES : estudio analitico en la industria textil para la vestimenta
}

\begin{abstract}
RESUMEN
Las investigaciones sobre los procesos comportamentales medioambientales representan temas privilegiados en el ámbito de las PyMES. Sin embargo, son difícilmente perceptibles ya que conllevan cambios de visiones, de valores, de actitudes y de intenciones. Por otra parte, sabemos muy poco sobre la intención de los dirigentes de las PyMES frente al medioambiente. El objetivo de este artículo es explorar la naturaleza y la pertinencia de las relaciones entre la intención medioambiental de los dirigentes y lo que las determinan. Esta investigación se basa en el ámbito del empresariado sostenible y moviliza la teoría del comportamiento de Ajzen (1991). Se ha realizado bajo una perspectiva de exploración a partir de una encuesta de veinte directores de PyMES del sector del textil para la vestimenta en Túnez y de un análisis léxico. Los resultados conducen a formular ocho propuestas relativas a los factores determinantes de la intención medioambiental. Ellos conciernen más precisamente a las consecuencias esperadas del comportamiento buscado, a las acciones de las empresas para realizarlos, a las presiones de los poderes públicos y los mandantes extranjeros, a las percepciones de las aptitudes medioambientales y de la disponibilidad de los recursos, a la naturaleza de la actividad y al deseo de imitar los modelos empresariales.
\end{abstract}

Palabras clave

Empresariado sostenible, Intención medioambiental, Responsabilidad social de las empresas, Sector textil para la vestimenta. Teoria del comportamiento planificado

\section{INTRODUCTION}

L'importance croissante des questions environnementales dans la vie des affaires incite à l'élaboration de stratégies entrepreneuriales appropriées (Cordano et Frieze, 2000). Dans cette perspective, les processus comportementaux de conception de stratégies durables représentent un terrain de recherche privilégié (Ivanaj et McIntyre, 2006). Toutefois, la connaissance du rôle de l'entrepreneur dans l'exploitation d'opportunités dans le domaine de l'environnement durable est assez peu développée (Dean et McMullen, 2007 ; York et Venkataraman, 2010). De même, l'influence des attitudes et des normes pro-environnementales sur le comportement environnemental demeure peu étudiée (Garcia-Valiñas, Macintyre et Torgler, 2012).

La littérature en management s'intéresse depuis une dizaine d'années aux déterminants du comportement responsable des dirigeants (Reynaud et al., 2008a). Toutefois, excepté des recherches importantes à l'instar de celles de Ajzen, Joyce, Sheikh et Cote (2011); Kuckertz et Wagner (2010) ; Martin-Pena, Diaz-Garrido et Sanchez-Lopez (2010) et Perrin (2011), nous en savons peu sur l'intention comportementale des managers à l'égard de l'environnement (Cordano et Frieze, 2000). Pourtant, il a été démontré dans des domaines aussi différents que la psychosociologie (Ajzen, 1991 ; 2002), le marketing, la gestion des ressources humaines et l'entrepreneuriat, le rôle majeur de l'intention dans la prédiction de différents types de com- 
portement. Une meilleure compréhension de la décision entrepreneuriale en PME suppose d'explorer la notion d'intention environnementale. Celle-ci est essentielle pour saisir les changements nécessaires menant vers une organisation écologique (Flannery et May, 2000). Comprendre les facteurs explicatifs de cette intention renseigne sur une phase majeure du processus comportemental environnemental.

L'objectif de cet article est de mieux comprendre les relations entre l'intention environnementale des dirigeants des PME et ses déterminants à partir d'une perspective exploratoire. Il ne s'agit pas de mesurer l'intensité et la validité de ces liens, mais de comprendre la nature et la pertinence des facteurs prédisant cette intention. Cette recherche s'ancre dans le champ de l'entrepreneuriat durable et s'appuie sur la théorie du comportement planifié. Pour mieux comprendre les déterminants de l'intention entrepreneuriale, nous avons mené une étude qualitative dans le secteur du textile-habillement en Tunisie à partir de vingt entretiens qui ont fait l'objet d'une analyse lexicale. Les résultats conduisent à la formulation de huit propositions relatives aux déterminants de l'intention environnementale.

Dans la première partie, nous présenterons notre ancrage théorique dans le champ de l'entrepreneuriat durable et de la théorie du comportement planifié de Ajzen (1991) et proposerons une définition de l'intention environnementale. Dans la deuxième partie, nous décrirons les déterminants de l'intention et formulerons des propositions. Le cadre méthodologique sera exposé dans la troisième partie. Nous présenterons ensuite les résultats de l'enquête selon leur pertinence par rapport à la littérature mobilisée. Nous engagerons enfin une discussion sur les résultats et reviendrons sur les propositions de façon synthétique. Nous concluons par les apports, les limites et les perspectives de la recherche.

\section{LES DIMENSIONS PSYCHOSOCIOLOGIQUES DE LENTREPRENEURIAT DURABLE}

Depuis les années 2000, le champ de l'entrepreneuriat durable connaît une dynamique croissante. Il peut bénéficier des avancées relatives aux modèles de décision entrepreneuriale inspirés de la théorie du comportement planifié. Après être revenus sur notre positionnement dans le champ de l'entrepreneuriat durable, nous proposons de définir l'intention environnementale et de montrer la contribution de la théorie du comportement planifié à mieux comprendre la formation de cette intention chez les dirigeants de PME.

\subsection{Positionnement de la recherche dans le champ de l'entrepreneuriat durable}

Notre recherche est positionnée dans le champ de l'entrepreneuriat durable ${ }^{1}$ par référence à Spence, Ben Boubaker Gherib et Ondou Biwolé (2011) qui considèrent l'engagement des PME

1 Les travaux de Cohen et Winn (2007) ; Pacheco, Dean et Payne (2010) et Shepherd et Patzelt (2011) fournissent une revue de littérature détaillée sur l'entrepreneuriat durable et des concepts connexes. 
dans le développement durable comme un acte entrepreneurial. Pour œuvrer à la construction de ce champ, Shepherd et Patzelt (2011) suggèrent deux questionnements : qu'est-ce qui doit être soutenu? Qu'est-ce qui doit être développé ? Les relations construites entre ces deux problématiques se font à travers l'action entrepreneuriale. S'inspirant de la littérature sur le développement durable et l'entrepreneuriat, ces auteurs affirment que le but de l'entrepreneuriat durable est de comprendre comment cette action contribue à la préservation de l'environnement naturel, c'est-à-dire la terre, la biodiversité et les écosystèmes. Ceci permet, en particulier dans les pays en développement, d'améliorer l'éducation, la productivité, la santé et l'autonomie des individus. La recherche en entrepreneuriat durable est donc nécessaire pour explorer le rôle de l'action entrepreneuriale en tant que mécanisme de soutien à la nature et aux écosystèmes tout en procurant des gains économiques et non économiques pour l'investisseur, l'entrepreneur et plus largement pour la société.

York et Venkataraman (2010) soutiennent que la responsabilité sociale de l'entreprise et le rôle de l'entrepreneur participent à l'émergence de l'entrepreneuriat durable en tant que champ théorique propre à l'étude des problèmes environnementaux. L'entrepreneuriat durable peut inclure des aspects de la responsabilité sociale de l'entreprise qui se réfère à des actions œuvrant pour le bien social, au-delà des intérêts de l'entreprise et des exigences de la loi. Pour Spence, Ben Boubaker Gherib et Ondou Biwolé (2011), l'entrepreneuriat durable est la volonté du dirigeant de PME d'intégrer dans les processus organisationnels des pratiques managériales durables prenant en compte les dimensions sociale et environnementale de la responsabilité sociale de l'entreprise. Au même titre que cette dernière, l'entrepreneuriat durable permet de comprendre la résolution de problèmes environnementaux (York et Venkataraman, 2010).

Pour les besoins de cette recherche, nous considérons l'entrepreneuriat durable comme un processus de poursuite d'opportunité qui se concrétise par des actions en vue de résoudre des problèmes environnementaux. Que leurs origines soient coercitives (à travers la pression exercée par les différentes parties prenantes), incitatives (grâce aux mesures des pouvoirs publics) ou le fruit d'une volonté individuelle, cette poursuite d'opportunité peut concerner les volets économique, social ou environnemental de la RSE. D'un point de vue théorique et expérimental, nous explorons l'intention de l'acte d'entreprendre spécifiquement à travers sa composante environnementale.

\subsection{De l'intention entrepreneuriale à l'intention environnementale}

Les actions sont constituées d'une composante physique et d'une composante mentale (Searle, 1980). L'intention s'inscrit au sein de cette dernière. Selon Ganascia (1996), « la plupart des activités cognitives sont conçues comme intentionnelles, en ce sens qu'elles répondent à une intention, c'est-à-dire à un but ». Pour Cossette (2004), « les gens agissent consciemment de façon intentionnelle en vue d'atteindre leurs buts ». En s'appuyant sur son étymologie latine, il est possible de définir l'intention comme la volonté de tendre vers un but.

L'intention est mobilisée pour étudier les comportements dans de nombreuses disciplines comme la psychologie, la gestion, la philosophie ou le droit. Pour Ajzen (1991), ce concept est censé saisir les facteurs motivationnels qui influencent le comportement. L'intention entre- 
preneuriale est une volonté personnelle (Tounés, 2006 ; Vesalainen et Pihkala, 1999) qui inclut les fins et les moyens (Krueger et Carsrud, 1993). Elle représente un processus cognitif qui naît avec les motivations, les valeurs et les croyances. Elle est fortement influencée par des variables situationnelles (Vesalainen et Pihkala, 1999).

Dans le domaine du développement environnemental, rares sont les définitions de l'intention ${ }^{2}$. D’après Hines, Hungerford et Tomera (1986), l'intention environnementale désigne la volonté d'agir ou de se comporter d'une certaine façon. Elle représente une interaction entre des variables cognitives (capacité d'actions, connaissances des stratégies et de leur aboutissement) et personnelles (attitudes, contrôle interne, responsabilité personnelle). L'intention environnementale se manifeste au moment où une personne déclare publiquement vouloir accomplir une action. Pour Martin-Pena, Diaz-Garrido et Sanchez-Lopez (2010), elle détermine la façon avec laquelle les managers répondent aux problématiques environnementales.

Nous définissons l'intention environnementale comme une phase majeure du processus cognitif de concrétisation d'actions entrepreneuriales en vue de résoudre des problèmes environnementaux. Elle est une volonté individuelle conditionnée par les contextes organisationnel, culturel, politique et économique. Elle peut être étudiée par le biais de la théorie du comportement planifié de Ajzen (1991).

\subsection{L’apport de la théorie du comportement planifié}

D'après Shepherd et Patzelt (2011), la psychologie est l'une des disciplines la plus représentée dans le champ de l'entrepreneuriat. La psychologie du développement durable joue un rôle crucial pour déplacer le monde vers des actions durables (Oskamp et Schultz, 2006). Au niveau individuel, cette discipline fournit des opportunités de recherche pour élargir la contribution de l'action entrepreneuriale au champ de l'entrepreneuriat durable.

Selon Martin-Pena, Diaz-Garrido et Sanchez-Lopez (2010), une approche psychologique fondée sur l'étude des processus d'influence de facteurs individuels et contextuels sur l'intention produit des résultats pertinents dans le domaine de l'entrepreneuriat durable. Cette approche est particulièrement appropriée pour la prédiction du comportement environnemental. Bien qu'elle soit peu mobilisée dans la prise de décision éthique (Flannery et May, 2000), la théorie du comportement planifié de Ajzen (1991) étudie avec succès l'intention environnementale (Kurland, 1995 ; Kuckertz et Wagner, 2010). Elle confère à l'intention une place centrale dans la genèse du comportement (Ajzen, 1991 ; 2002). Cette théorie peut servir de point de départ pour analyser la relation entre l'intention et le comportement (Martin-Pena, Diaz-Garrido et Sanchez-Lopez, 2010). Elle prédit celui-ci par le biais de trois groupes de facteurs : l'attitude vis-à-vis du comportement souhaité, les normes subjectives et le contrôle comportemental perçu (Ajzen, 1991 ; 2002).

2 Il est surprenant de noter que dans le numéro spécial de l'Academy of Management Journal, deux articles dédiés à ce sujet ne définissent pas ce concept (Cordano et Frieze, 2000 ; Flannery et May, 2000). 
Après avoir montré l'ancrage de la recherche dans le champ de l'entrepreneuriat durable ainsi que l'intérêt de la théorie du comportement planifié, nous proposons d'analyser plus finement les déterminants de l'intention environnementale.

\section{LES DÉTERMINANTS DE LINTENTION ENVIRONNEMENTALE}

Pour comprendre les antécédents de l'intention environnementale des dirigeants de PME, nous formulons trois groupes de propositions liées aux attitudes associées au comportement, aux normes subjectives et au contrôle comportemental perçu.

\subsection{Les attitudes associées au comportement environnemental}

Des individus sensibilisés aux problèmes environnementaux manifestent de plus fortes attitudes environnementales (Torgler et García-Valiñas, 2007). Celles-ci déterminent l'engagement responsable des dirigeants (Reynaud et al., 2008a). Selon Cordano et Frieze (2000), différents travaux confirment l'influence significative des attitudes environnementales sur l'intention des managers de prévenir la pollution. D’après Flannery et May (2000), cellesci exercent un effet positif sur l'intention de ces derniers de traiter des eaux usées. Pour Kuckertz et Wagner (2010), des attitudes favorables au développement durable influencent l'intention entrepreneuriale des étudiants.

Selon Gergen, Gergen et Jurtas (1992), les trois composantes de l'attitude sont cognitives, affectives et conatives. Nous nous intéressons particulièrement aux deux dernières catégories $^{3}$. La composante affective repose sur l'appréciation du comportement recherché. Pour Habhab-Rave (2008), les bénéfices espérés de ce dernier sont "une conséquence tout de même suffisamment intéressante pour expliquer que la vaste majorité des PME déjà impliquée dans l'environnement avait l'intention, soit de poursuivre leur engagement, soit de l'augmenter ». La quête du profit, d'une meilleure image (Bansal et Roth, 2000 ; Del Brio et Junquera, 2003b) ou d'une stratégie de différenciation (Hamdoun, 2008) sont des avantages recherchés à travers le management environnemental.

La composante conative se manifeste par les actions engagées pour concrétiser l'intention. Adopter un comportement environnemental est un processus cognitif tourné vers l'action. Selon Vallerand (1994), les attitudes guident vers cette dernière. L'intention est facilement réalisée si un plan d'exécution est développé (Schwartz, 1992). La responsabilité environnementale des managers requiert l'initiation d'une série d'actions afin de réduire la pollution de leurs activités (Bansal et Roth, 2000). Les actions entreprises par les dirigeants peuvent

3 Selon ces auteurs, ces trois catégories sont interactives, de sorte qu'une modification de l'une entraîne des changements sur les autres. La dimension cognitive implique que l'individu possède des croyances ou opinions sur le comportement souhaité. Celles-ci interviennent dans le processus de formation du sentiment (favorable ou défavorable) relatif à ce dernier. Nous considérons que ces croyances et opinions sont intégrées dans la catégorie affective. 
être la recherche d'informations auprès des parties prenantes, l'élaboration de cahiers des charges et le montage de dossiers de subvention. Ainsi, en référence à la théorie d'Ajzen (1991), nous formulons deux propositions :

P1.a : les conséquences positives espérées du comportement souhaité influencent positivement l'intention environnementale des dirigeants.

P1.b : les actions entreprises en vue de concrétiser le comportement souhaité influencent positivement l'intention environnementale des dirigeants.

\subsection{Les normes subjectives}

Dans le champ de la responsabilité sociale, l'évolution vers des pratiques industrielles écologiques est considérée selon les intérêts, les valeurs et les motivations des parties prenantes (Fineman et Clarke, 1996). La réalisation d'un comportement environnemental est critique, car il implique la coopération de plusieurs groupes d'intérêts (Cordano et Frieze, 2000). Selon la théorie des parties prenantes (Freeman, 1984), les pressions de ces groupes conduiraient les managers à adopter des comportements davantage respectueux de l'environnement. La littérature sur l'environnement identifie principalement quatre types de parties prenantes (Fineman et Clarke, 1996 ; Henriques et Sadorsky, 1999) : les parties prenantes de régulation, les parties prenantes organisationnelles, les parties prenantes communautaires et les médias. L'identification et les perceptions de ces dernières déterminent les réponses des dirigeants de l'industrie aux pressions qu'elles exercent sur eux (Fineman et Clarke, 1996). Les perceptions de ces pressions diffèrent selon les profils (proactifs versus réactifs) de l'engagement environnemental des dirigeants (Henriques et Sadorsky, 1999).

Selon Ajzen (1991), les normes reflètent nos perceptions de ces pressions à l'égard du comportement que nous ciblons. Ces normes représentent l'importance que nous accordons à l'approbation des parties prenantes et à notre volonté (contrainte) de s'y conformer. Certaines pressions extérieures sont très contraignantes pour les entreprises exerçant des activités polluantes (Del Brio et Junquera, 2003b). L'effet associé des influences sociales, juridiques et organisationnelles contraint les comportements environnementaux des dirigeants (Martin-Pena, Diaz-Garrido et Sanchez-Lopez, 2010).

À l'opposé de la contrainte, l'influence des parties prenantes est davantage perçue comme une opportunité par les entrepreneurs ayant l'intention d'obtenir la certification ISO 14001 par rapport à ceux qui n'en ont pas (Del Brio et Junquera, 2003b). Selon l'approche par les ressources (Pfeffer et Salancik, 1978), certaines sociétés de l'industrie chimique considèrent que la réglementation est un avantage concurrentiel, car elles ont les moyens pour s'adapter et d'internaliser les coûts générés (Fineman et Clarke, 1996). Abstraction faite de la nature des pressions (contrainte versus opportunité), nous nous intéressons à la relation entre l'intention environnementale et les perceptions des managers de l'importance des pressions des parties prenantes externes. Nous proposons que :

P2.a : les pressions des parties prenantes externes influencent positivement l'intention environnementale des dirigeants. 
Les parties prenantes internes n'ont pas une vision commune de l'importance que devrait accorder leur entreprise aux enjeux environnementaux. Toutefois, les salariés sont des agents qui ont une forte influence sur la réussite ou l'échec de toute initiative environnementale (Henriques et Sadorsky, 1999). Ils jouent un rôle important dans l'engagement environnemental de leur firme (Ramus et Steger, 2000 ; Del Brio, Fernandez et Junquera, 2007). Potentiellement, ils sont en mesure d'exercer des pressions pro-environnementales sur leur propre industrie (Fineman et Clarke, 1996). Ainsi, nous formulons la proposition suivante :

P2.b : les pressions des parties prenantes internes influencent positivement l'intention environnementale des dirigeants.

Pour mieux comprendre les activités environnementales, il faut analyser l'ancrage culturel de l'entrepreneur au sein de son milieu social, mais aussi ses caractéristiques et ses valeurs. Dans le champ de l'entrepreneuriat durable, notent Meek, Pacheco et York (2010), les normes subjectives renvoient aux systèmes de valeurs individuelles. Si celles-ci sont en accord avec l'écologie, alors ces normes influencent la probabilité d'exploiter une opportunité dans le secteur environnemental. D’après Reynaud et al. (2008a ; 2008 b), cette probabilité est fortement déterminée par la culture et le pays dans lequel vit l'entrepreneur. En effet, à travers leur comparaison internationale, il s'avère que des individus davantage individualistes, accordent une importance plus faible aux responsabilités environnementale et sociale par rapport à ceux qui sont plus collectivistes. La supériorité des valeurs altruistes est à rapprocher d'une plus forte prise en compte de la responsabilité sociale de l'entreprise dans les pays avec un haut niveau de développement économique.

L'entrepreneur est davantage enclin aux changements stratégiques compatibles avec ses valeurs et perceptions de l'écologie (Bansal et Roth, 2000). Selon Flannery et May (2000), les obligations morales des dirigeants de l'industrie américaine du métal influencent leur intention éthique. Ces derniers entreprennent davantage de mesures environnementales quand ils sont sensibilisés aux défis de l'environnement. Plus forte est cette sensibilisation, meilleure est leur intention (Del Brio et Junquera, 2003b). Nous nous proposons d'examiner la nature de l'influence des valeurs culturelles sur l'intention :

P2.c : les valeurs culturelles des dirigeants influencent positivement leur intention environnementale.

\subsection{Le contrôle comportemental perçu}

Selon Ajzen (1991), le contrôle comportemental correspond à la facilité perçue pour réaliser un comportement donné. L'auteur distingue des facteurs de contrôle internes et externes. Les premiers renvoient aux compétences entrepreneuriales de l'individu. Selon Hungerford et Volk (1990), un savoir-faire environnemental a un impact direct sur l'adoption d'un comportement durable. Un individu est davantage porté vers l'action s'il maîtrise le problème auquel il est confronté. Pour Flannery et May (2000), les compétences et les capacités des managers sont primordiales pour mettre en place un dispositif environnemental. L'intention environnementale requiert la perception d'aptitudes facilitant le comportement. Dans ce cadre, la formation a un impact significatif sur l'engagement environnemental (GarciaValiñas, Macintyre et Torgler, 2012 ; Torgler et García-Valiñas, 2007). 
Par ailleurs dans le secteur de l'industrie, l'expérience passée est déterminante pour surmonter les obstacles liés à la gestion de nouveaux projets (Politis, 2005). Cette expérience contribue à la survie et à la croissance des firmes industrielles (Gimeno, Folta, Cooper et Woo, 1997). Des expériences similaires dans le domaine de l'environnement, affirment Martin-Pena, Diaz-Garrido et Sanchez-Lopez (2010), peuvent être déterminantes sur les perceptions des stratégies organisationnelles du dirigeant. Plus particulièrement, le secteur du textile-habillement tunisien manque de compétences humaines et génère des pollutions exigeant un savoir-faire confirmé. Nous formulons ainsi deux propositions :

P3.a : les perceptions des aptitudes environnementales acquises grâce à des formations spécialisées influencent positivement l'intention environnementale des dirigeants.

P3.b : les perceptions des aptitudes environnementales acquises par le biais des expériences passées influencent positivement l'intention environnementale des dirigeants.

Les facteurs de contrôle comportemental externes sont contextuels. Pour Sharma (2000) et Aragon-Correa et Sharma (2003), les dirigeants construisent activement le futur s'ils contrôlent judicieusement l'environnement. Selon Meek, Pacheco et York (2010), les incitations financières influencent l'engagement environnemental des entreprises industrielles. D'après Del Brio et Junquera (2003a ; 2003b), l'intention environnementale requiert des managers aux compétences appropriées. Par ailleurs, les perceptions de la facilité d'accès aux informations, conseils et accompagnement peuvent agir sur l'intention (Olufunso, 2010 ; Tounés, 2006).

Selon Henriques et Sadorsky (1999), les réseaux sociaux (informels) sont une source d'informations importantes. Pour Bansal et Roth (2000), ils facilitent l'engagement des entreprises dans des activités écologiques. D’après Spence, Ben Boubaker Gherib et Ondou Biwolé (2011), ils procurent des compétences uniques pour les PME s'engageant dans des stratégies de développement durable. D’après Cordano et Frieze (2000), le soutien de collègues au sein d'associations peut aider les managers à réduire les effets polluants de leurs exploitations. L'implication dans des structures environnementales augmente la préférence des individus à protéger l'environnement (Garcia-Valiñas, Macintyre et Torgler, 2012 ; Torgler et García-Valiñas, 2007). Celle-ci permet aux dirigeants de nouer des liens avec leurs pairs en vue de les soutenir dans leurs démarches environnementales. Nous suggérons ces deux propositions :

P4.a : les perceptions de la disponibilité des ressources (informations, conseils, accompagnement, finances et ressources humaines) influencent positivement l'intention environnementale des dirigeants.

P4.b : l’implication des dirigeants dans des réseaux sociaux écologiques influence positivement leur intention environnementale.

À partir de cette revue de littérature sur l'entrepreneuriat durable, la psychologie du développement durable et la responsabilité sociale, nous avons formulé trois groupes de propositions pour comprendre les déterminants de l'intention environnementale. Nous allons exposer le protocole de recherche déployé pour vérifier la pertinence de ces propositions. 


\section{PROPOSITION D'UN CADRE MÉTHODOLOGIQUE}

Les recherches sur le processus d'engagement environnemental sont en nombre faible. Celles réalisées concernent essentiellement des populations européennes et américaines (Starik et Marcus, 2000) et sont expérimentées au sein des grandes entreprises. Hors, les principes régissant la durabilité dans les pays du nord ne sont pas nécessairement valables pour les PME des pays du sud (Spence, Ben Boubaker Gherib et Ondou Biwolé, 2011). En Tunisie, l'état des connaissances actuelles sur ce processus est pauvre. Excepté les travaux de Ben Boubaker Gherib et Berger-Douce (2008) et Spence, Ben Boubaker Gherib et Ondou Biwolé (2007 ; 2011), aucune étude n'est réalisée sur ce dernier. Ainsi, une méthodologie exploratoire est appropriée pour étudier la nature et la pertinence des facteurs prédisant l'intention des dirigeants des PME tunisiennes.

Pour contextualiser l'intention au sein de ce pays, cette méthodologie est adéquate pour étudier en profondeur des différences situationnelles (Wacheux, 1996). Dans cette perspective, celle-ci mobilise l'entretien semi-directif. Celui-ci est le moyen d'investigation le plus utilisé dans les recherches en sciences de gestion (Gavard-Perret, Gotteland, Haon et Jolibert, 2008). Il « est l'outil de prédilection de la phase exploratoire d'une enquête dans la mesure où... il est lui-même un processus exploratoire... Les entretiens exploratoires ont pour fonction de mettre en lumière les aspects du phénomène auxquels le chercheur ne peut penser spontanément et de compléter les pistes de travail suggérées par ses lectures » (Blanchet et Gottman, 1992).

Nous ciblons les entreprises industrielles du secteur du textile-habillement du Sahel tunisien. Nous avons choisi un seul secteur, car les enjeux environnementaux sont assez homogènes (Flannery et May, 2000) et les incidences écologiques sont semblables (Bansal et Roth, 2000). Les défis environnementaux de ce secteur sont particulièrement importants. D'une part, les matériaux et les procédés de production utilisés sont fortement polluants. D’autre part, celles-ci sont fortement soumises aux exigences de normalisation des pouvoirs publics et des clients étrangers.

Nous avons réalisé notre enquête durant le deuxième trimestre 2010. Nous avons mené des interviews semi-directives en face-à-face avec les dirigeants. Celles-ci duraient entre $45 \mathrm{mi}$ nutes et 75 minutes. Elles ont été enregistrées puis retranscrites au fur et à mesure. Une fiche synthétique reprenant les principales données est rédigée pour chaque entretien. Explicitons la cible de l'étude, l'outil d'investigation et les techniques d'analyse mobilisées.

\subsection{L'échantillon de l'étude}

En Tunisie, le nombre d'entreprises du secteur du textile-habillement est de 2036, soit $36 \%$ de l'ensemble des firmes manufacturières nationales. Celles employant 10 personnes et plus représentent plus de $40 \%$ de l'effectif total de l'industrie manufacturière, soit 198280 emplois $^{4}$.

4 Données recueillies au mois de juin 2011 sur le site Internet de l'Agence de promotion de l'industrie et de l'innovation. 
Le Sahel est la zone géographique qui recèle le plus grand nombre d'entreprises du secteur en Tunisie, soit 889 ( $44 \%$ des firmes nationales du textile-habillement).

Nous avons utilisé la base de données de l'Agence nationale de protection de l'environnement (ANPE). Nous avons fait une requête sur les entreprises accompagnées par cet organisme dans la réalisation de démarches environnementales. Celles appartenant à un groupe étranger ont été exclues, car l'entreprise mère peut être l'instigatrice des stratégies durables. Au final, la requête est composée de 148 firmes réparties entre Sousse (87), Monastir (52) et Mahdia (9). Parmi elles, 37 ont répondu favorablement à notre sollicitation, soit $25 \%$. Seuls 20 des 37 dirigeants ont l'intention de mettre en place dans un avenir plus ou moins proche une mesure ou une politique environnementale. Conscients que les résultats sont limités dans leur généralisation, nous avons tout de même opéré de multiples relances téléphoniques et électroniques. Il n’a pas été possible d'augmenter le nombre de répondants. Les activités les plus représentées sont la confection, la teinture, la sérigraphie et le délavage. La décomposition des entreprises selon leurs caractéristiques est reproduite dans le tableau 1.

TABleau 1. CARACtéRISTIQUeS DESCRIPTIVES DE L’ÉCHANTILLON

\begin{tabular}{|c|c|c|c|c|}
\hline PME & $\begin{array}{l}\text { Année } \\
\text { création/ } \\
\text { Reprise }\end{array}$ & Activité & Effectif & International $^{5}$ \\
\hline $\mathbf{A}$ & 1949 & Teinture étoffée en maille & 180 & T.E \\
\hline B & 1970 & Housses, tapis-moquettes et autres produits & 65 & P.E \\
\hline $\mathbf{C}$ & 1980 & $\begin{array}{l}\text { Articles pour bébés, maillots de bain, vêtements } \\
\text { pour enfants }\end{array}$ & 220 & P.E \\
\hline D & 1985 & $\begin{array}{l}\text { Fabrication de produits chimiques pour } \\
\text { l'industrie textile }\end{array}$ & 145 & N.E \\
\hline $\mathbf{E}$ & 1986 & Emballage et teinture & 100 & T.E \\
\hline $\mathbf{F}$ & 1990 & Teinture, délavage, sérigraphie et broderie & 30 & T.E \\
\hline $\mathbf{G}$ & 1990 & Délavage & 100 & T.E \\
\hline $\mathbf{H}$ & 1993 & Confection de corsets, maillots et sous-vêtements & 132 & T.E \\
\hline I & 1995 & Teinture et délavage de jeans & 50 & T.E \\
\hline $\mathbf{J}$ & 1995 & $\begin{array}{l}\text { Confection de robes, jupes et vêtements pour } \\
\text { enfants }\end{array}$ & 70 & T.E \\
\hline $\mathbf{K}$ & 1995 & $\begin{array}{l}\text { Traitement sur articles confectionnés, teinture } \\
\text { délavage et sérigraphie }\end{array}$ & 86 & P.E \\
\hline $\mathbf{L}$ & 1999 & $\begin{array}{l}\text { Traitements sur articles confectionnés, teinture, } \\
\text { délavage et sérigraphie }\end{array}$ & 70 & T.E \\
\hline M & 1999 & Confection de maillots de bain et sous-vêtements & 105 & P.E \\
\hline
\end{tabular}

5 Légende : T.E : totalement exportatrice; P.E : partiellement exportatrice ; N.E : non-exportatrice. 


\begin{tabular}{lllcl}
\hline $\mathbf{N}$ & 1999 & Production de tissus & 180 & T.E \\
\hline $\mathbf{O}$ & 2000 & Confection & 220 & T.E \\
\hline $\mathbf{P}$ & 2002 & $\begin{array}{l}\text { Finissage, produits de traitement sur étoffes } \\
\text { tissées : blanchissement, teinture, impression }\end{array}$ & 59 & T.E \\
\hline $\mathbf{Q}$ & 2004 & $\begin{array}{l}\text { Traitements sur articles confectionnés, teinture, } \\
\text { délavage et sérigraphie }\end{array}$ & 54 & T.E \\
\hline $\mathbf{R}$ & 2004 & Confection et délavage & 150 & T.E \\
\hline $\mathbf{S}$ & 2005 & $\begin{array}{l}\text { Confection de prêt-à-porter, lavage et traitement } \\
\text { spéciaux }\end{array}$ & 110 & T.E \\
\hline $\mathbf{T}$ & 2006 & $\begin{array}{l}\text { Traitements sur articles confectionnés (teinture, } \\
\text { délavage et sérigraphie) }\end{array}$ & 100 & P.E \\
\hline
\end{tabular}

\subsection{Outil et techniques d'investigation}

Le guide d'entretien, repris dans le tableau 2, s'articule autour de trois thèmes. La colonne de gauche reprend les groupes de propositions dans l'ordre croissant de leurs énoncés. La colonne de droite indique les questions afférentes à ces derniers.

L'analyse de contenu est majoritairement déployée pour identifier les attitudes et les intentions des dirigeants (Gavard-Perret et al., 2008). Pour comprendre en profondeur la thématique de l'intention, nous avons opéré une analyse de contenu lexicale (Chenitz et Swanson, 1986). Celle-ci « repose sur le postulat que la répétition d'éléments de discours (mots, expressions ou significations similaires) révèle les centres d'intérêt et les préoccupations des acteurs » (Thietart, 2003).

Les données collectées sont traitées grâce à l'analyse fréquentielle d'apparition des mots de manière isolée ou en association avec d'autres mots. L'élément de discours est l'unité d'analyse. Grâce au logiciel Sphinx, nous comptabilisons les répétitions des mots et des expressions clés. Cette technique permet d'interpréter les discours des dirigeants à travers la nature et la richesse du vocabulaire déployé. En recourant aux grilles de dépouillement et aux fréquences d'apparition, nous croisons l'analyse verticale (entretien par entretien) et l'analyse horizontale (thème par thème), présentons les résultats en reprenant des éléments de discours illustrant la pertinence (et non pas la validité) des concepts préalablement mobilisés.

\section{ANALYSE DES RÉSULTATS}

L'objectif de cette étude n'est pas de confirmer la validité des propositions précédemment élaborées. Nous cherchons à montrer la pertinence de facteurs expliquant l'intention environnementale des dirigeants des PME tunisiennes du textile-habillement. Nous interprétons les discours selon l'ordre croissant des propositions formulées. 


\begin{tabular}{cc}
\hline Variables/Propositions & Questions \\
\hline Attitudes vis-à-vis du comportement &
\end{tabular}

\section{Attitudes vis-à-vis du comportement}

P1.a : les conséquences positives espérées 9. Quels sont les résultats que vous du comportement souhaité influencent espérez réaliser en adoptant une démarche positivement l'intention environnementale des environnementale ?

dirigeants.

1. Aviez-vous initié des actions pour P1.b : les actions entreprises en vue de concrétiser votre intention d'adopter cette concrétiser le comportement souhaité démarche?

influencent positivement l'intention 2. Est-ce que vous avez eu cette intention environnementale des dirigeants.

\section{Les normes subjectives} environnementale suite à une volonté personnelle, pour imiter des concurrents, P2.a : les pressions des parties prenantes groupes de personnes, organisations externes à l'entreprise influencent positivement publiques ou politiques ?

l'intention environnementale des dirigeants. 3. Quels sont ces groupes de personnes P2.b : les pressions des parties prenantes et organisations qui ont influencé votre internes à l'entreprise influencent positivement intention ?

l'intention environnementale des dirigeants. 6. Votre ouverture à l'international a-t-elle influencé votre intention d'adopter des mesures ou des politiques environnementales?

4. Avez-vous eu l'intention de mettre

P2.c : les valeurs culturelles des dirigeants influencent positivement leur intention environnementale.

en place des mesures ou des politiques environnementales pour des raisons culturelles, sociales, politiques, et/ou économiques?

\section{Le contrôle comportemental perçu}

P3.a : les perceptions des aptitudes environnementales acquises grâce à des formations spécialisées influencent positivement l'intention environnementale des dirigeants.

P3.b : les perceptions des aptitudes environnementales acquises par le biais des expériences passées influencent positivement l'intention environnementale des dirigeants.

P4.a : les perceptions de la disponibilité des ressources (informations, conseils, accompagnement, finances et ressources humaines) influencent positivement l'intention environnementale des dirigeants.

P4.b : l'implication des dirigeants dans des réseaux sociaux écologiques influence positivement leur intention environnementale. 


\subsection{Les facteurs pertinents}

L'attitude vis-à-vis du comportement environnemental souhaité est analysée par les avantages attendus (P1.a) et les actions entreprises pour le concrétiser (P1.b). Les attentes du dirigeant sont fondamentales dans le processus de mise en œuvre de pratiques de protection de l'environnement (Cordano et Frieze, 2000). D'après le dirigeant de l'entreprise E, « Il est évident que les avantages potentiels sont invisibles... » Dans le tableau 3, le traitement des discours renseigne que l'amélioration de la légitimité et de l'image vis-à-vis des pouvoirs publics, des donneurs d'ordres étrangers et des institutions financières (20 occurrences) et une amélioration des conditions de travail (6 occurrences) priment sur la réduction des coûts (6 occurrences) et l'augmentation des parts de marché (5 occurrences). Les conséquences espérées à travers l'intention environnementale sont davantage non pécuniaires.

TABLEAU 3. EXPRESSIONS-CLÉS RELATIVES AUX RÉSULTATS ESPÉRÉS

\begin{tabular}{lc}
\hline & Fréquence \\
\hline Amélioration de l'image vis-à-vis des organismes publics & 7 \\
\hline Amélioration de la légitimité vis-à-vis des organismes publics & 7 \\
\hline Amélioration des conditions de travail & 6 \\
\hline Augmentation des ventes, des clients & 5 \\
\hline Amélioration de l'image vis-à-vis des clients étrangers & 4 \\
\hline Réduction des coûts de production & 4 \\
\hline Amélioration de la légitimité vis-à-vis des institutions financières & 2 \\
\hline Réduction des coûts de consommation énergétiques & 2 \\
\hline & Total \\
\hline
\end{tabular}

D'après Cordano et Frieze (2000), les recherches mobilisant la théorie du comportement planifié intègrent des items pour évaluer les actions entreprises en vue de réaliser l'intention comportementale. Le tableau 4 met en évidence que la recherche d'informations est l'action la plus significative dans l'explication de cette dernière ( 22 occurrences). Les fournisseurs d'équipements, les organismes spécialisés et les patrons du secteur sont les cibles privilégiées. Le dirigeant de la firme $\mathrm{D}$ déclare que «pour l'acquisition de la station de traitement des eaux, j'ai commencé par la recherche des fournisseurs des équipements nécessaires ». Le patron de l'entreprise R affirme qu'il a chargé ses ingénieurs de «chercher les fournisseurs ayant des prix d'équipements les moins chers".

L'ANPE et l'ONAS sont sollicités pour des conseils sur les dossiers de subvention d'investissement. Le dirigeant de l'entreprise $\mathrm{N}$ déclare que « je me suis renseigné (auprès de l'ANPE) sur la formation pour la mise en place de la certification ISO 14001, le coût généré et les subventions disponibles ». Dans le même temps, 7 dirigeants ont monté des dossiers relatifs à l'entreprise de démarches environnementales dans le but de bénéficier des aides de l'ANPE et de l'ONAS. Deux d'entre eux ont mis en œuvre des plans en vue d'engager ces démarches.

6 Dans ce tableau et les suivants, le total peut être supérieur à 20, car un dirigeant peut évoquer plus d'une idée ou d'un mot-clé. 
Avoir un plan environnemental et formaliser un document décrivant ce dernier dénotent l'engagement durable de l'entrepreneur (Henriques et Sadorsky, 1999).

TABleau 4. EXPressions-CLÉS AFFÉrentes AUX ACTIONS ENTREPRiSeS

\begin{tabular}{|c|c|}
\hline & Fréquence \\
\hline Recherche d'informations auprès des équipementiers & 11 \\
\hline Recherche d'informations auprès d'organismes spécialisés & 7 \\
\hline Préparation d'un dossier pour l'ANPE et l'ONAS & 7 \\
\hline Recherche d'informations auprès des dirigeants du même secteur & 4 \\
\hline Allocation de personnel & 3 \\
\hline Préparation d'un nouveau plan & 2 \\
\hline \multirow{2}{*}{ Formation des salariés } & 2 \\
\hline & Total \\
\hline
\end{tabular}

Dans le cadre de notre enquête, la pertinence des normes subjectives dans l'explication de l'intention est appréciée par les pressions des parties prenantes externes (P2.a), internes (P2.b) et des valeurs culturelles des dirigeants (P2.c). Le croisement des analyses horizontale et verticale (tableau 5) montre la détermination des pressions des pouvoirs et des organismes publics dans la formation de l'intention environnementale de ces derniers (9 occurrences). Parmi les dirigeants se conformant aux recommandations publiques, celui de la firme $\mathrm{C}$ annonce que «l'ANPE a exigé l'achat de cette station pour améliorer la qualité de l'eau rejetée dans l'environnement ». Le dirigeant de l'entreprise I déclare que "l'ANPE nous a recommandé dès le démarrage de l'activité d'installer une station de traitement des eaux vu la dangerosité des substances chimiques utilisées".

Pour le dirigeant de l'entreprise J, «mon intention est devenue plus intense lorsque j'ai contacté les responsables de l'ANPE qui m'ont vivement conseillé d'installer cette station pour se prémunir contre les pénalités et les amendes ». La peur des sanctions financières (8 occurrences) contrainte à vouloir mettre en place une démarche environnementale. Les exigences de certification et de normalisation des donneurs d'ordres européens (6 occurrences) sont aussi pertinentes pour comprendre l'intention environnementale des dirigeants d'entreprises opérant totalement ou partiellement à l'export. D'après celui de l'entreprise $\mathrm{G}$, " la démarche de se certifier ISO 14001 est une exigence des clients étrangers... Les clients renommés exigent la certification environnementale». Le dirigeant de l'entreprise K tient un discours similaire ; en effet, "les clients étrangers les plus connus dans le jean choisissent des sous-traitants certifiés... Pour cette raison, nous allons essayer d'avoir ISO $14001 »$. Enfin, les dirigeants souhaitent s'aligner sur les dispositifs environnementaux mis en place par les concurrents (5 occurrences).

Les perceptions des aptitudes acquises grâce à des formations spécialisées (P3.a) et des expériences antérieures (P3.b) contribuent de manière pertinente à la détermination de l'intention environnementale. Les formations sont jugées nécessaires par 4 dirigeants dans l'acquisition des aptitudes environnementales (tableau 6). Selon le dirigeant de l'entreprise D, «des formations accélérées dans le domaine environnemental " sont nécessaires pour augmenter les aptitudes de l'ensemble du personnel. Le patron de la firme T déclare que « Ce qui a faci- 
lité mon engagement sont... les programmes de formation en management environnemental que j'ai eu lors d'une précédente expérience ».

TABLEAU 5. EXPRESSIONS-ClÉS CONCERNANT LES NORMES SUBJECTIVES

\begin{tabular}{lc}
\hline & Fréquence \\
\hline Se prémunir des amendes et contraventions & 8 \\
\hline Sous contraintes des organismes environnementaux (ONAS et ANPE) & 7 \\
\hline Sous contraintes des clients étrangers & 6 \\
\hline Pour s'aligner sur la concurrence & 5 \\
\hline Sous contraintes des pouvoirs publics (États et collectivités locales) & 2 \\
\hline Sur demande du personnel & 1 \\
\hline & Total \\
\hline
\end{tabular}

De même, quatre entrepreneurs confirment le bénéfice de l'expérience dans le domaine du textile sur leurs intentions. Pour le dirigeant de l'entreprise $\mathrm{M}$, "l'expérience passée en tant que salarié dans une entreprise de confection est bénéfique... J’ai une idée sur les pratiques environnementales pour réduire les coûts de production». Celui de la firme $\mathrm{P}$ affirme que " mon expérience en tant que codirigeant d'une entreprise de textile a un impact direct... Je connais les étapes de préparation du dossier de subvention et les fournisseurs des stations".

Le dernier groupe de facteurs déterminant de façon pertinente l'intention environnementale regroupe les perceptions de l'accessibilité aux différentes ressources (P4.a). Celles-ci contribuent de manière importante à la formation de cette intention. La disponibilité des moyens financiers est la principale ressource citée par les dirigeants d'entreprises (11 occurrences). Bénéficier des informations, du conseil et de l'accompagnement des structures spécialisées accélère le processus cognitif intentionnel de 9 dirigeants. Le dirigeant de l'entreprise J déclare que "l'existence des subventions de l'État a renforcé mon intention ». L'accès aux subventions publiques pour acquérir des stations de traitement des eaux explique de manière pertinente l'intention environnementale de 6 dirigeants. Le temps est un « input » nécessaire pour 4 managers ayant l'intention de se certifier ISO 14001.

TAbleau 6. Expressions-ClÉs Relatives au Contrôle Comportemental PERÇU

\begin{tabular}{lc}
\hline & Fréquence \\
\hline Existence de moyens financiers & 11 \\
\hline Subventions de l'ANPE & 6 \\
\hline Le manque de temps & 4 \\
\hline Accès aux informations et conseils & 5 \\
\hline Expériences antérieures dans le domaine & 4 \\
\hline Accompagnement par l'ANPE & 4 \\
\hline Formations spécialisées & 4 \\
\hline Compétences du personnel & 2 \\
\hline
\end{tabular}




\subsection{Les facteurs non pertinents et les facteurs générés}

Les analyses de discours renseignent que les pressions des employés ne contribuent pas à l'explication du processus d'engagement environnemental des dirigeants du textile-habillement tunisien. 19 entrepreneurs n'accordent aucun intérêt à ces pressions dans leur processus intentionnel. De même, les aspects culturels, plus précisément religieux, n’ont pas de poids pertinent dans l'explication de l'intention environnementale. Hormis son impact sur 2 dirigeants, la religion ne joue pas un rôle fondamental dans la formation de cette dernière. Selon le patron de l'entreprise $\mathrm{B}$, «notre religion n'a rien à avoir avec l'adoption des actions environnementales. À mon avis, la religion défend plutôt des questions d'ordre social ». Pour le dirigeant de l'entreprise D, «peu importe la religion, aujourd'hui les questions environnementales conditionnent l'existence de l'entreprise et sa croissance».

L'investigation révèle que les réseaux sociaux ne sont pas un support d'entraide dans le processus de construction de stratégies durables. En Tunisie, il n'existe pas de structures et d'associations exclusivement dédiées aux thématiques environnementales. D’après le patron de la firme $\mathrm{C}$, «dans notre région, on n'a pas de réseaux qui s'intéressent aux questions écologiques ». Seuls 2 dirigeants sont impliqués dans des structures professionnelles. Celles-ci n'étant pas spécialisées dans l'environnement, ils ne trouvent pas un espace de mutualisation des compétences. En effet, le patron de l'entreprise E annonce que « je suis membre de la jeune chambre économique, j'assiste parfois aux réunions, mais on n'aborde pas les questions d'ordre écologique ». Celui de la firme J déclare qu'il «assiste souvent aux réunions de l'UTICA (Union tunisienne de l'industrie, du commerce et de l'artisanat), mais on n'a jamais traité des sujets relatifs à l'environnement ou à la nécessité de le protéger».

La nature de l'activité explique pertinemment la formation de l'intention environnementale des dirigeants des PME tunisiennes. Ceux spécialisés dans le délavage, la teinture et la sérigraphie entreprennent un plus grand nombre de mesures ou de politiques environnementales (19). Ces dernières concernent davantage le recyclage des eaux usées (12) (tableau 7). Les enjeux environnementaux de ces types d'activités sont plus importants que ceux de la confection à cause de la dangerosité et du volume des produits toxiques polluant l'eau utilisée dans le processus de fabrication. Le patron de l'entreprise G déclare que "le style de notre activité génère des pollutions énormes... Ceci renforce notre volonté d'installer une station de traitement des eaux». Pour le patron de la firme R, "je n'ai pas eu de pressions internes ou externes, le type de notre activité provoque des pollutions d'air qui peuvent gêner le personnel, ainsi que des rejets liquides qui nuisent à l'environnement externe». Selon le dirigeant de l'entreprise B, «personne ne m'a obligé à recycler les déchets ou acheter des casques pour mes salariés... Notre activité génère trop de bruit et de déchets et nous contraint à recycler et à acheter les casques».

L'étude empirique montre le souhait de 5 dirigeants (entreprises $\mathrm{H}, \mathrm{J}, \mathrm{N}, \mathrm{O}$ et $\mathrm{S}$ ) d'imiter des entrepreneurs ayant adopté des pratiques environnementales. Le patron de la firme $\mathrm{H}$ affirme que "j'ai imité des collègues avec qui j'entretiens des relations. Ils mont bien conseillé et guidé pour la préparation du dossier de subvention de l'ANPE ». Selon le dirigeant de l'entreprise J, "j'ai eu l'intention d'installer la station d'eau juste pour imiter les dirigeants les plus renommés de mon domaine». Pour le patron de la firme $\mathrm{S}$, « en installant 
une station de traitement des eaux, j'ai essayé d'imiter mes concurrents... S'ils ont procédé à cela, c'est parce qu'ils attendent des avantages".

Tableau 7. Mesures ou politiques environnementales SElon la NatUre de L'ACTIVITÉ

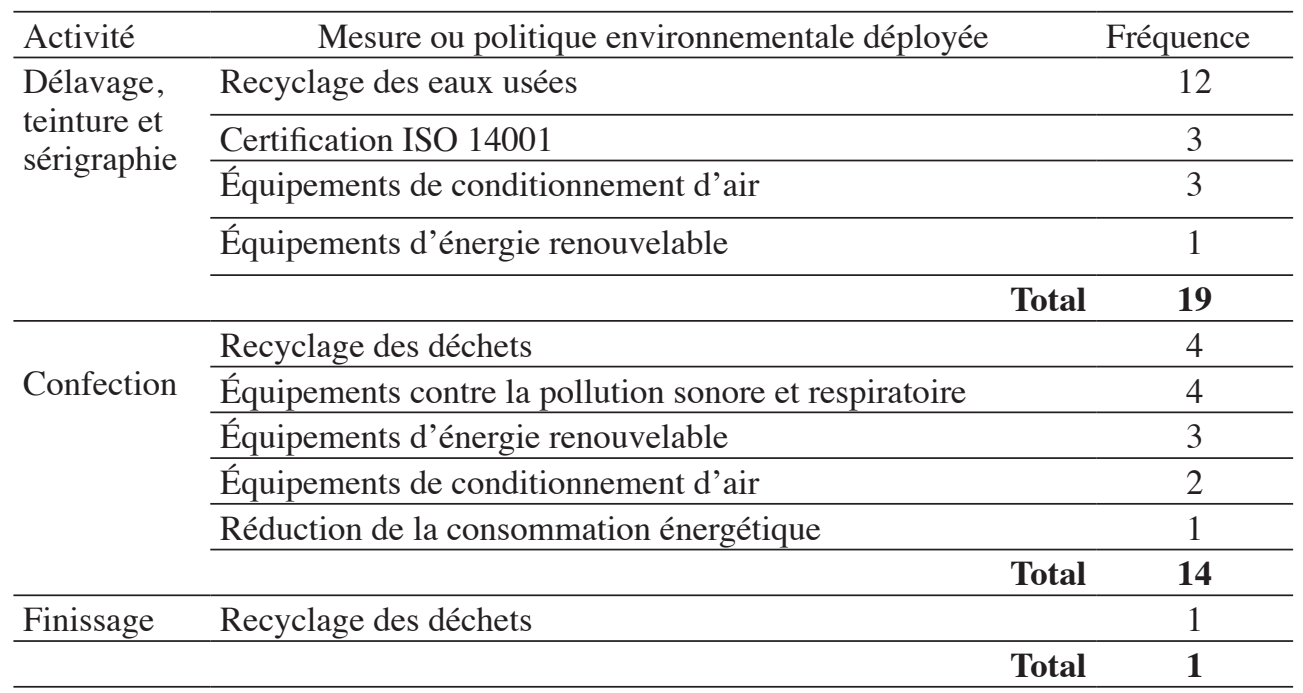

\section{DISCUSSION DES RÉSULTATS}

Selon Kuckertz et Wagner (2010), les recherches en entrepreneuriat durable n'intègrent pas conséquemment les corpus théoriques, conceptuels et empiriques nécessaires à l'étude des comportements, des intentions, des attitudes et des normes sociales. Déployer les principales théories de l'entrepreneuriat pour expliquer des thématiques de développement durable enrichit davantage les modèles conceptuels de ce champ. Des attitudes et des normes sociales environnementales sont très pertinentes pour comprendre l'engagement des individus dans la protection de l'environnement (Garcia-Valiñas, Macintyre et Torgler, 2012). Bien qu'elle soit peu employée en entrepreneuriat durable, la théorie du comportement planifié est utilisée avec réussite pour étudier l'intention environnementale (Cordano et Frieze, 2000 ; Flannery et May, 2000). Ajzen et al. (2011) confirment qu'elle prédit avec succès l'intention environnementale des étudiants dans le domaine de la conservation de l'énergie. Située dans le cadre de l'industrie du textile-habillement du Sahel tunisien, notre recherche conforte la robustesse de cette théorie.

Les résultats de l'étude indiquent que les propositions P1.a, P1.b, P2.a, P3.a, P3.b et P4.a sont pertinentes pour prédire l'intention environnementale et s'accordent avec les travaux mobilisés. Les conséquences positives espérées du comportement recherché par les dirigeants d'entreprises (P1.a) sont déterminantes dans la formation de cette intention. 
Celles-ci sont davantage non pécuniaires confirmant ainsi les conclusions de Kuckertz et Wagner (2010). La recherche de la compétitivité n'est pas un facteur majeur de l'engagement durable des entrepreneurs tunisiens (Spence, Ben Boubaker Gherib et Ondou Biwolé, 2011). Ceux-ci sont en quête d'une meilleure image et d'une plus forte légitimité à l'égard des pouvoirs publics. Pour ceux dont l'activité est totalement ou partiellement destinée à l'international, cette quête est motivée à l'égard des donneurs d'ordres étrangers. Enfin, les dirigeants d'entreprises souhaitent offrir de meilleures conditions de travail à leurs salariés. Cette proposition s'accorde avec les conclusions de Pedersen (2010). En effet, les managers de firmes industrielles européennes et américaines perçoivent que la sécurité au travail, la santé et le développement personnel des employés sont des responsabilités prioritaires de l'entreprise.

En Tunisie, Ben Boubaker Gherib et Berger-Douce (2008) déplorent un déficit cruel d'informations tant en termes de réglementation environnementale que de dispositifs d'aide destinés aux PME. Ceci expliquerait vraisemblablement que les actions entreprises par les patrons pour concrétiser le comportement souhaité (P1.b) se focalisent essentiellement sur la recherche d'informations auprès des différentes parties prenantes. Selon Martin-Pena, Diaz-Garrido et Sanchez-Lopez (2010), l'intention environnementale se traduit par une série d'actions définissant les objectifs et les stratégies environnementales. Collecter de l'information permet d'éclairer les dirigeants dans le processus cognitif comportemental. Associer aux demandes de conseils pour le montage de dossiers de subvention auprès de l'ANPE et de l'ONAS, cette collecte est pertinente dans l'explication de cette intention.

L'intention d'adopter une mesure environnementale se manifeste souvent en réaction à la réglementation et aux pressions des parties prenantes de régulation (P2.a) confirmant ainsi les conclusions de Bansal et Roth (2000) ; Cordano et Frieze (2000); Sharma (2000). Sans une réglementation environnementale appropriée au secteur de l'industrie, les comportements des dirigeants britanniques n’auraient probablement pas changé (Fineman et Clarke, 1996). «La motivation réglementaire est, pour les PME comme pour les grandes entreprises, la première motivation pour intégrer des préoccupations sociales ou environnementales dans le management " (Quairel et Auberger, 2005). Éviter les sanctions et les amendes contraint le management environnemental des firmes (Del Brio et Junquera, 2003b).

Les donneurs d'ordres étrangers sont une partie prenante organisationnelle (Fineman et Clarke, 1996 ; Henriques et Sadorsky, 1999) qui exerce d'importantes pressions pour le respect des normes de production internationale. En raison d'une forte dépendance au commerce extérieur (Spence, Ben Boubaker Gherib et Ondou Biwolé, 2011), l'export joue un rôle majeur dans l'engagement environnemental des dirigeants tunisiens (Ben Boubaker Gherib et Berger-Douce, 2008). En effet, la certification et la normalisation occupent une place importante dans le management environnemental des dirigeants d'entreprises (Martin-Pena, Diaz-Garrido et Sanchez-Lopez, 2010 ; Meek, Pacheco et York, 2010).

Les perceptions des aptitudes développées grâce à des formations spécialisées contribuent favorablement à l'explication de l'intention environnementale. Ainsi, la proposition P3.a conforte la recherche de Spence, Ben Boubaker Gherib et Ondou Biwolé (2007) sur l'importance de la formation des employés dans l'engagement des PME tunisiennes dans des pratiques de développement durable. Selon Flannery et May (2000), les compétences des managers sont décisives dans la réalisation de démarches environnementales. De même, des 
expériences antérieures dans le domaine de l'environnement sont pertinentes pour prédire cette intention. Selon ces auteurs, celles-ci augmentent la confiance et le niveau de cette dernière. Également, les perceptions de disponibilité des moyens financiers, des informations et des conseils, l'accompagnement d'organismes spécialisés et un personnel compétent (P4.a) sont des facteurs judicieux pour comprendre l'intention. En effet, d'après York et Venkataraman (2010), la disponibilité d'informations et des incitations financières encouragent l'adoption de dispositifs luttant contre la dégradation de l'environnement.

À l'opposé, les investigations renseignent que trois propositions ne sont pas pertinentes pour prédire l'intention environnementale des dirigeants tunisiens. À l'instar de l'enquête de Fineman et Clarke (1996), les salariés n'ont pas un rôle pertinent dans la formation de cette dernière (P2.b). Une explication possible serait les relations autoritaires entre la hiérarchie et les salariés. Ainsi, dans la culture tunisienne, ces derniers ne s'impliquent pas dans les enjeux environnementaux de leurs firmes de peur de perdre leurs emplois. Une deuxième explication serait la faiblesse du rôle des syndicats dans le secteur industriel concerné.

La religion n'explique pas la formation de l'intention environnementale des dirigeants (P2.c) confirmant de la sorte des travaux antérieurs réalisés au Maghreb. Ben Oumlil et Balloun (2009) confirment que le culte musulman a un effet mitigé, voire faible, sur l'intention éthique des managers marocains. Plus précisément en Tunisie, les convictions religieuses jouent un faible rôle dans le déclenchement des activités en faveur de l'environnement (Spence, Ben Boubaker Gherib et Ondou Biwolé, 2011). A contrario, nos résultats ne s'accordent pas avec les travaux réalisés en Europe et en Amérique du Nord. L'influence des normes sociales sur les comportements pro-environnementaux diffère selon les contextes et les populations étudiées (Flannery et May, 2000 ; Reynaud et al., 2008a). Enfin, l'impact de l'implication des dirigeants dans des réseaux écologiques sur l'intention n'est pas conforme à la littérature déployée sur la Tunisie pour soutenir la proposition P4.b. En effet, selon Spence, Ben Boubaker Gherib et Ondou Biwolé (2011), les entreprises intègrent des réseaux stratégiques pour acquérir des ressources et des connaissances limitant les risques de leur engagement durable. Ce résultat ne corrobore pas non plus avec ceux de Bansal et Roth (2000) portant sur des firmes britanniques et japonaises et de Spence, Ben Boubaker Gherib et Ondou Biwolé (2007) appliqué à des PME québécoises.

Les entretiens avec les dirigeants d'entreprises ont mis en évidence deux facteurs pertinents qui sont insuffisamment ou pas examinés dans notre revue de littérature. Le premier concerne la nature de l'activité. Celle-ci explique pertinemment la formation de l'intention environnementale confortant ainsi les déterminants de l'engagement environnemental des dirigeants tunisiens (Ben Boubaker Gherib et Berger-Douce, 2008). Au Canada, les champs d'activité semblent aussi discriminer les comportements environnementaux dans l'industrie des hydrocarbures (Sharma, 2000). Les stratégies entrepreneuriales des firmes se démarquent selon qu'elles sont spécialisées en amont ou en aval de la chaine de production.

Le deuxième facteur est relatif au désir d'imiter des entrepreneurs ayant réussi dans des démarches environnementales. Les modèles d'entrepreneurs ont une influence importante sur la décision d'un porteur de projet (Van Auken, Fry et Stephens, 2006 ; Meek, Pacheco et York, 2010). Ils affectent les intentions entrepreneuriales en changeant les attitudes, les 
capacités et les croyances. Les pairs influencent le système de valeurs et les perceptions de désirabilité des individus (Shapero et Sokol, 1982). " La perception sociale est également influencée par le contexte dans lequel on observe les actions d'autrui... Ceux qui sont déjà engagés malgré l'ambiguïté de la situation » (Gergen, Gergen et Jurtas, 1992). Confrontés à des problématiques éthiques, les individus s'identifient de manière importante à des modèles de référence pour décider de leur comportement (Rajeev, 2012). Ainsi, l'analyse des discours permet de formuler deux nouvelles propositions :

P5 : la nature de l'activité influence positivement l'intention environnementale des dirigeants.

P6 : la connaissance de modèles d'entrepreneur et le souhait de les imiter influencent positivement l'intention environnementale des dirigeants.

Tableau 8. Synthèse de LA PeRTinence des Propositions

\begin{tabular}{|c|c|c|c|}
\hline \multicolumn{2}{|c|}{ Propositions } & Idées dominantes & Occurrences \\
\hline \multirow{11}{*}{$\begin{array}{l}\text { Attitudes } \\
\text { vis-à-vis du } \\
\text { comportement }\end{array}$} & \multirow{7}{*}{$\begin{array}{l}\text { P1.a : conséquences } \\
\text { espérées du } \\
\text { comportement } \\
\text { souhaité }\end{array}$} & Résultat non économique & 26 \\
\hline & & 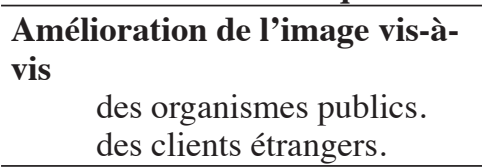 & \\
\hline & & $\begin{array}{l}\text { Amélioration de la légitimité } \\
\text { vis-à-vis } \\
\text { des organismes publics. } \\
\text { des institutions financières. }\end{array}$ & \\
\hline & & $\begin{array}{l}\text { Amélioration des conditions de } \\
\text { travail }\end{array}$ & \\
\hline & & Résultat économique & 11 \\
\hline & & $\begin{array}{l}\text { Réduction des coûts } \\
\text { de production. } \\
\text { de la consommation } \\
\text { énergétique. } \\
\end{array}$ & \\
\hline & & $\begin{array}{l}\text { Augmentation du chiffre } \\
\text { d'affaires }\end{array}$ & \\
\hline & \multirow[t]{4}{*}{$\begin{array}{l}\text { P1.b : actions } \\
\text { entreprises en vue } \\
\text { de concrétiser le } \\
\text { comportement } \\
\text { souhaité }\end{array}$} & $\begin{array}{l}\text { Recherche d'informations } \\
\text { auprès } \\
\text { des équipementiers. } \\
\text { d'organismes spécialisés. } \\
\text { des dirigeants du même } \\
\text { secteur. } \\
\end{array}$ & 22 \\
\hline & & $\begin{array}{l}\text { Montage de dossiers de } \\
\text { subvention }\end{array}$ & 7 \\
\hline & & $\begin{array}{l}\text { Formation et allocation des } \\
\text { salariés }\end{array}$ & 5 \\
\hline & & Mise en ouvre de plans & 2 \\
\hline
\end{tabular}




\begin{tabular}{|c|c|c|c|}
\hline \multirow[t]{3}{*}{$\begin{array}{l}\text { Les normes } \\
\text { subjectives }\end{array}$} & \multirow[t]{2}{*}{$\begin{array}{l}\text { P2.a : pressions des } \\
\text { parties prenantes } \\
\text { externes }\end{array}$} & $\begin{array}{l}\text { Contraintes } \\
\text { des pouvoirs } \\
\text { publics (organismes } \\
\text { environnementaux, État et } \\
\text { collectivités locales). } \\
\text { des clients étrangers. } \\
\text { des concurrents. } \\
\end{array}$ & 20 \\
\hline & & Échapper aux amendes & 8 \\
\hline & $\begin{array}{l}\text { P6 : connaissance } \\
\text { de modèles } \\
\text { d'entrepreneur }\end{array}$ & $\begin{array}{l}\text { Imitation de modèles } \\
\text { d'entrepreneur }\end{array}$ & 5 \\
\hline \multirow{4}{*}{$\begin{array}{l}\text { Le contrôle } \\
\text { comportemental } \\
\text { perçu }\end{array}$} & \multirow{2}{*}{$\begin{array}{l}\text { P3.a et P3.b : } \\
\text { perceptions des } \\
\text { aptitudes }\end{array}$} & Formation & 4 \\
\hline & & Expérience & 4 \\
\hline & \multirow{2}{*}{$\begin{array}{l}\text { P4.a : perceptions de } \\
\text { la disponibilité des } \\
\text { ressources }\end{array}$} & $\begin{array}{l}\text { Disponibilité des ressources } \\
\text { fonds propres. } \\
\text { subventions publiques. } \\
\text { temps. } \\
\text { compétence. } \\
\end{array}$ & 23 \\
\hline & & $\begin{array}{l}\text { Accès aux informations et } \\
\text { conseils et à l'accompagnement }\end{array}$ & 9 \\
\hline \multirow[t]{2}{*}{$\begin{array}{l}\text { Nature } \\
\text { de l'activité }\end{array}$} & \multirow[t]{2}{*}{$\begin{array}{l}\text { P5 : nature de } \\
\text { l'activité }\end{array}$} & $\begin{array}{l}\text { Délavage, teinture et } \\
\text { sérigraphie }\end{array}$ & 12 \\
\hline & & Confection & 5 \\
\hline
\end{tabular}

Nous proposons de synthétiser les déterminants de l'intention environnementale selon la pertinence des propositions. Le tableau 8 présente les idées dominantes par ordre décroissant d'occurrence, en distinguant trois groupes homogènes de variables. Notre étude met en exergue trois niveaux d'analyse dans la prédiction de l'intention environnementale : individuel, organisationnel et interorganisationnel. Une PME est dirigée par un dirigeant (individuel), fonctionne avec et pour ses salariés (organisationnel), et est en relation avec d'autres entreprises et institutions publiques tout en étant ancrée dans son environnement naturel (interorganisationnel). Identifier et gérer le rôle de chaque acteur à chaque niveau est essentiel pour atteindre la durabilité. L'association de ces trois grilles de lecture laisse envisager des perspectives théoriques multidisciplinaires (Bansal et Roth, 2000). L'entrepreneuriat durable apparaît ainsi comme un champ nécessitant des approches économiques, institutionnelles et sociologiques (Meek, Pacheco et York, 2010).

\section{CONCLUSION}

Notre recherche contribue à clarifier le processus intentionnel menant au comportement environnemental (Flannery et May, 2000 ; Martin-Pena, Diaz-Garrido et Sanchez-Lopez, 2010). Sur un plan théorique, comprendre la nature, et non pas l'intensité et la significa- 
tion statistique, des liens entre l'intention environnementale d'un côté, les attitudes, les normes sociales et le contrôle comportemental perçu de l'autre, s'est révélé particulièrement intéressant. Il ressort de cette étude que si la volonté personnelle est prépondérante pour saisir l'intention, elle doit également être située dans un contexte organisationnel, culturel, politique et économique.

Cette recherche aboutit à une contribution managériale dans la mesure où elle offre aux décideurs politiques un outil d'intervention adapté. Disposer d'une meilleure connaissance des déterminants de l'intention environnementale des dirigeants contribue à améliorer les dispositifs de réduction des effets néfastes des activités industrielles sur l'environnement et sur l'homme. Cette étude facilite la conception d'un accompagnement adapté aux problématiques de traitement des eaux, des déchets de la teinture, de l'impression et du délavage des tissus. Pour susciter l'intention environnementale, il est nécessaire d'intervenir en amont des activités par la sensibilisation des dirigeants de PME sur les défis environnementaux de l'industrie tunisienne du textile-habillement. Il ne s'agit pas seulement de communiquer sur la conformité à la législation, mais sur les bénéfices de ces défis pour la compétitivité des entreprises (Bansal et Roth, 2000 ; Martin-Pena, DiazGarrido et Sanchez-Lopez, 2010).

Les entrepreneurs indiquent que l'action publique doit faciliter l'accès aux ressources financières, informationnelles et humaines. Elle doit favoriser le montage de formations spécialisées dans le domaine environnemental. Il serait pertinent de soutenir les dirigeants d'entreprise par la création d'espaces de dialogue. Rencontrer des managers ayant réussi permet également d'améliorer le niveau de l'intention. L'un des principaux enjeux de l'entrepreneuriat durable ne semble pas être l'acquisition de connaissances managériales, mais plutôt le développement de l'inspiration entrepreneuriale à travers la diffusion d'histoires de réussites de PME (Kuckertz et Wagner, 2010).

Bien qu'offrant un cadre approprié pour expliquer l'intention environnementale, cette recherche comporte deux limites. La première est inhérente au décalage temporel entre l'intention et le comportement. La perspective intentionnelle n'est pas une condition nécessaire et suffisante à l'aboutissement du processus comportemental environnemental. Elle rend compte d'une photographie à un moment précis et dans un contexte donné. Même si elle "est soumise à une exigence de stabilité »(Bratman, 1997), le déphasage chronologique entre l'intention et le comportement pourrait empêcher ce dernier de se concrétiser. Toute intention, même suffisamment forte ne peut garantir la réalisation de l'acte. D'après Kuckertz et Wagner (2010), le passage à l'acte peut ainsi avoir lieu des mois, voire des années après.

La deuxième limite est relative au protocole de recherche. Les données recueillies ne permettent pas de se prononcer sur la fiabilité et la validité des propositions, mais seulement sur leur pertinence. Un projet de plus grande envergure permettra de tester le pouvoir explicatif de ces propositions. À partir d'un cadre hypothético-déductif, nous examinerons leur validité prédictive auprès d'un échantillon représentatif d'entreprises du secteur du textile-habillement en Tunisie.

Pour des raisons évidentes d'homogénéité de la population interrogée, les déterminants de l'intention environnementale mis en évidence sont appropriés pour le secteur étudié. Leur pertinence n'est pas représentative de l'ensemble des secteurs concernés par des enjeux 
environnementaux. Il sera opportun d'explorer les spécificités de chaque secteur considéré afin d'enrichir notre connaissance de l'intention environnementale. L'étude de cette variable permet certes de comprendre pourquoi des managers entreprennent, mais surtout pourquoi ils choisissent des activités créatrices de richesses sociétales (Meek, Pacheco et York, 2010). Outre la particularité sectorielle, les propositions développées pourraientelles s'appliquer à toute taille d'entreprise ? Les résultats d'études empiriques conduisent à des résultats divergents. Contrairement à la situation observée à Québec (Spence, Ben Boubaker Gherib et Ondou Biwolé, 2007), l'étude de Ben Boubaker Gherib et BergerDouce (2008) montre l'absence d'effet taille sur l'engagement environnemental des PME tunisiennes. Même si la réalité des PME est différente de celle des grandes entreprises, la taille semblerait avoir une influence mitigée sur le processus d'engagement durable. Ce résultat nous invite à mener de nouvelles investigations en privilégiant une approche contingente.

\section{RÉFÉRENCES}

AJZen, I. (1991). The theory of planned behaviour. Organizational Behaviour and Human Decision Process, 50(2), 179-211.

AJZEN, I. (2002). Residual effects of past on later behavior : habituation and reasoned action perspectives. Personality and Social Psychology Review, 6(2), 107-122.

Ajzen, I., Joyce, N., Sheikh, S. et Cote, N.G. (2011). Knowledge and the prediction of behavior : the role of information accuracy in the theory of planned behavior. Basic and applied social psychology, 33(2), 101-117.

Aragon-Correa, A. et Sharma, S. (2003). A contingent resource-based view of proactive corporate environmental strategy. Academy of Management Review, 28(1), 71-88.

BANSAL, P. et Roth, K. (2000). Why companies do green : a model of ecological responsiveness. Academy of Management journal, 43(4), 717-736.

Ben Boubaker Gherib, J. et Berger-Douce, S. (2008, mai). L'engagement environnemental des $P M E$ : une analyse comparative France-Tunisie. Conférence de l'Association internationale de management stratégique. Nice, France.

Ben Oumlil, A. et BAlloun, J.L. (2009). Ethical decision-making differences between American and Moroccan managers. Journal of Business Ethics, 84(4), 457-478.

Blanchet, A. et Gottman, A. (1992). L'Enquête et ses méthodes : l'entretien. Paris, Nathan.

Bratman, M.E. (1997). Intention partagée et obligation mutuelle. Dans J.P. Dupuy et P. Livet (dir.), Les limites de la rationalité: rationalité, éthique et cognition (p. 246-266). Paris, La Découverte.

Chenitz, W.C. et SWANSON, J. (1986). From practice to grounded theory : qualitative research in nursing. Californie, Addison-Wesley.

Cohen, B. et Winn, M.I. (2007). Market imperfections, opportunity and sustainable entrepreneurship. Journal of Business Venturing, 22(1), 29-49.

Cordano, M. et Frieze, I.H. (2000). Pollution reduction preferences of US environmental managers : applying Ajzen's theory of planned behavior. Academy of Management Journal, 43(4), 627-641. 
Cossette, P. (2004). L'organisation, une perspective cognitiviste. Québec, Presses de l’Université Laval.

Dean, T.D. et McMullen, J.S. (2007). Toward a theory of sustainable entrepreneurship : Reducing environmental degradation through entrepreneurial action. Journal of Business Venturing, 22(1), 5076.

Del Brio, J.A. et Junquera, B. (2003a). A review of the literature on environmental innovation management in SMEs : implication for public policies. Technovation, 32(12), 939-948.

Del Brio, J.A. et Junquera, B. (2003b). Influence of the perception of the external environmental pressures on obtaining the ISO 14001 standard in Spanish industrial companies. International Journal of Production Research, 41(2), 337-348.

Del Brio, J.A., Fernandez, E. et Junquera, B. (2007). Management and employee involvment in achieving an environmental action-based competitive advantage : an empirical study. The International Journal of Human Resource Management, 18(4), 491-522.

Fineman, S. et Clarke, K. (1996). Green stakeholders : industry interpretations and response. Journal of Management Studies, 33(6), 715-730.

Flannery, B.L. et May, D.R. (2000). Environmental ethical decision making in the U.S. metal-finishing industry. Academy of Management Journal, 43(4), 642-662.

Freeman, R.E. (1984). Strategic management : a stakeholder approach. Pitman Series in Business and Public Policy.

Ganascia, J.G. (1996). Les sciences cognitives. Paris, Flammarion.

Garcia-Valiñas, M.A., Macintyre, A. et Torgler, B. (2012). Volunteering, pro-environmental attitudes and norms. The Journal of Socio-Economics, 41(4), 455-467.

Gavard-Perret, M-L., Gotteland, D., Haon, C. et Jolibert, A. (2008). Méthodologie de la recherche : réussir son mémoire ou sa thèse en science de gestion. Paris, Pearson Education.

Gergen, K.J., Gergen, M.M. et Jurtas, S. (1992). Psychologie sociale. Québec, Études Vivantes Éditions.

Gimeno, J., Folta, T.B., Cooper, A.C. et Woo, C.Y. (1997). Survival of the fittest ? Entrepreneurial human capital and the persistence of underperforming firms. Administrative Science Quarterly, 42(4), 750-783.

НАвнав-RAVE, S. (2008). Les dirigeants face à l'environnement: comment réconcilier environnement naturel et opportunités stratégiques ? $5^{\mathrm{e}}$ Colloque de l'Association pour le développement de l'enseignement et de la recherche sur la responsabilité sociale de l'entreprise. Grenoble.

Hamdoun, M. (2008). Le comportement écologique des entreprises chimiques tunisiennes : un passage obligé ou un choix délibéré. $17^{e}$ Conférence de l'Association internationale de management stratégique. Nice.

Henriques, I. et Sadorsky, P. (1999). The relationship between environmental commitment and managerial perceptions of stakeholder importance. Academy of Management Journal, 42(1), 87-99.

Hines J.M., Hungerford, H.R. et Tomera, A.N. (1986). Analysis and synthesis of research on responsible pro-environmental behaviour : a meta-analysis. The Journal of Environmental Education, $18(2), 1-8$.

Hungerford, H.R. et Volk, T.L. (1990). Changing learner behavior through environmental education. The Journal of Environmental Education, 21(3), 8-21. 
IvanaJ, V. et McIntyre, J. (2006, octobre). Multinational enterprises and sustainable development : a review of strategy process research. Communication au Colloque international «Multinational Enterprise and Sustainable Development: Strategic Tool for Competitiveness ». Atlanta, Georgie, États-Unis.

KRUeger, N.F. et CARSRUd, A.L. (1993). Entrepreneurial intentions : applying the theory of planned behaviour. Entrepreneurship and Regional Development, 5(4), 315-330.

Kuckertz, A. et Wagner, M. (2010). The influence of sustainability orientation on entrepreneurial intentions - Investigating the role of business experience. Journal of Business Venturing, 25(5), 524539.

Kurland, N.B. (1995). Ethical intentions and the theories of reasoned action and planned behavior. Journal of Applied Social Psychology, 25(4), 297-313.

Martín-Pena, M.L., Diaz-Garrido, E. et Sanchez-Lopez, J.M. (2010). Relation between management's behavioural intentions toward the environment and environmental actions. Journal of Environmental Planning and Management, 53(3), 297-315.

Meek, R.W., Pacheco, D.F. et York, J.G. (2010). The impact of social norms on entrepreneurial action : evidence from the environmental entrepreneurship context. Journal of Business Venturing, 25(5), 493-509.

Osкамp, S. et Schultz, P.W. (2006). Using psychological science to achieve ecological sustainability. Dans S. Donaldson, D. Berger et K. Pezdek (dir.), Applied psychology : new frontiers and rewarding careers (p. 81-106). Mahwah, NJ, Lawrence Erlbaum.

Olufunso, F.O. (2010). Graduate entrepreneurial intention in South Africa : motivations and obstacles. International Journal of Business and Management, 5(9), 87-98.

Pacheco, D.F., Dean, T.J. et Payne, D.S. (2010). Escaping the green prison : entrepreneurship and the creation of opportunities for sustainable development. Journal of Business Venturing, 25(5), 464-480.

Pedersen, E.R. (2010). Modelling CSR : how managers understand the responsibilities of business towards society. Journal of Business Ethics, 91(2), 155-166.

Perrin, J.L. (2011). Emotional responses to environmental messages and future behavioral intentions. Applied Environmental Education \& Communication, 10(3), 146-157.

Pfeffer, J. et Salancik, G.R. (1978). The external control of the organizations. New York, Harpers and Row.

Politis, D. (2005). The process of entrepreneurial learning : a conceptual framework. Entrepreneurship Theory and Practice, 29(4), 399-424.

Quairel, F. et Auberger, M.N. (2005). Management responsable et PME : une relecture du concept de responsabilité sociétale de l'entreprise. Revue des sciences de gestion, 40(211-212), 111-126.

RAJEEv, P.N. (2012). Correlates of ethical intentions : a critical review of empirical literature and suggestions for future research. Journal of International Business Ethics, 5(1), 3-17.

Ramus, C.A. et Steger, U. (2000). The roles of supervisory support behaviors and environmental policy in employee « ecoinitiatives » at leading-edge european companies. Academy of Management Journal, 43(4), 605-626.

Reynaud, E., Egri, C.P., Ralston, D.A, Palmer, I., Srinvasan, H., Fu, P., Butt, A., Gutierrez, J.R., Danis, W., Hoon, C.L., Yong-lin, M., Furrer, O., Pla-Barber, J., Molteni, M., Dabic, M., Bing, C., Kuo, C., Casado, T., De la Garza, T., Richards, M., Sidani, Y., Hallinger, P. et Milton, L. (2008a). Les déterminants du comportement responsable: une comparaison internationale à grande 
échelle. $17^{\mathrm{e}}$ Congrès de l'Association internationale de management stratégique, Université de Nice Sophia-Antipolis.

Reynaud, E., Egri, C.P., Ralston, D.A., Danis, W., Starkus, A., Dabic, M., Wangenheim, F., Dalgic, T., Castro, F., Ptocan, V.V., Kavoossi, M., Molteni, M., Girson, I., Elenkov, D., Pla-Barber, J. et Weber, M. (2008b). La responsabilité sociale de l'entreprise à l'épreuve de l'Europe. Revue française de gestion, 23(180), 109-130.

Schwartz, S.H. (1992). Universals in the content and structure of values : theoretical advances and empirical tests in 20 countries. Dans M. Zanna (dir.), Advances in experimental social psychology (p. 1-65). New York, Academic Press.

Searle, J.R. (1980). Minds, brains, and programs. Behavioral and Brain Sciences, 3(3), 417-424.

Shapero, A. et Sokol, L. (1982). The social dimensions of entrepreneurship. Dans C.A. Kent, D.L. Sexton, K.H. Vesper (dir.), Encyclopedia of entrepreneurship (p. 72-90). Englwood Cliffs, Prentice Hall.

Sharma, S. (2000). Managerial interpretations and organizational context as predictors of corporate choice of environmental strategy. Academy of Management Journal, 43(4), 681-697.

Shepherd, D.A. et Patzelt, H. (2011). The new field of sustainable entrepreneurship : studying entrepreneurial action linking what is to be «sustained » with « what is to be developed ». Entrepreneurship Theory and Practice, 35(1), 137-163.

Spence, M., Ben Boubaker Gherib, J. et Ondou Biwolé, V. (2007). Développement durable et PME : une étude exploratoire des déterminants de leur engagement. Revue internationale PME, 20(34), 18-42.

Spence, M., Ben Boubaker Gherib, J. et Ondou Biwolé, V. (2011). Sustainable entrepreneurship : is entrepreneurial will enough ? A North-South comparison. Journal of Business Ethics, 99(3), 335-367.

Starik, M. et Marcus, A. (2000). Introduction to the special research forum on the management of organizations in the natural environment : a field emerging from multiple paths, with many challenges ahead. Academy of Management Journal, 43(4), 539-546.

Thietart, R.A. (2003), Méthodes de recherche en management. Paris, Dunod.

Torgler, B. et García-Valiñas, M.A. (2007). The determinants of individuals' attitudes towards preventing environmental damage. Ecological Economics, 63(2-3), 536-552.

Tounés, A. (2006). L'intention entrepreneuriale des étudiants : le cas français, Revue des Sciences de Gestion, 3(219), 57-65.

Vallerand, J. (dir.) (1994). Les fondements de la psychologie sociale. Boucherville, Gaëtan Morin.

Van Auken, H., Fry, F.L. et Stephens, P. (2006). The influence of role models on entrepreneurial intentions. Journal of Developemental Entrepreneurship, 11(2), 157-167.

Vesalainen, J. et Pihkala, T. (1999). Motivation structure and entrepreneurial intentions. Dans P. Reynolds, W.D. Bygrave, S. Manigart, C.M. Mason, G.D. Meyer, H.J. Sapienza et K.J. Shaver (dir.), Frontiers of entrepreneurship research (p. 73-87). Wellesley, MA, Babson College.

Wacheux, F. (1996). Méthodes qualitatives et recherche en gestion. Paris, Économica.

York, J.C. et Venkataraman, S. (2010). The entrepreneur-environement nexus : uncertainty, innovation and allocation. Journal of Business Venturing, 25(5), 449-463. 